\title{
Oral symptoms and salivary findings in oral lichen planus, oral lichenoid lesions and stomatitis
}

\author{
Kristine Roen Larsen ${ }^{1 *}$, Jeanne Duus Johansen², Jesper Reibel ${ }^{1}$, Claus Zachariae ${ }^{3}$, Kasper Rosing ${ }^{4}$ \\ and Anne Marie Lynge Pedersen ${ }^{1}$
}

\begin{abstract}
Background: To examine if patients with oral lichen planus, oral lichenoid lesions and generalised stomatitis and concomitant contact allergy have more frequent and severe xerostomia, lower unstimulated and chewingstimulated saliva and citric-acid-stimulated parotid saliva flow rates, and higher salivary concentration of total protein and slgA than cases without contact allergy and healthy controls.

Methods: Forty-nine patients (42 women, aged $61.0 \pm 10.3$ years) and 29 healthy age- and gender-matched subjects underwent a standardised questionnaire on general and oral health, assessment of xerostomia, clinical examination, sialometry, mucosal biopsy and contact allergy testing.

Results: Nineteen patients had oral lichen planus, 19 patients had oral lichenoid lesions and 11 patients had generalised stomatitis. 38.8\% had contact allergy. Xerostomia was significantly more common and severe in patients (46.9\%) than in healthy controls, whereas the saliva flow rates did not differ. The patients had higher slgA levels in unstimulated and chewing-stimulated saliva than the healthy controls. The total protein concentration in saliva was lower in the unstimulated saliva samples whereas it was higher in the chewing stimulated saliva samples from patients when compared to healthy controls. The differences were not significant and they were irrespective of the presence of contact allergy.

Conclusion: Xerostomia is prevalent in patients with oral lichen planus, lichenoid lesions and generalised stomatitis, but not associated with salivary gland hypofunction, numbers of systemic diseases or medications, contact allergy, age, or gender. Salivary slgA levels were higher in patients than in healthy controls, but did not differ between patient groups. The total salivary protein concentration was lower in unstimulated saliva samples and higher in chewing-stimulated saliva samples in patients than in healthy controls, but did not differ between patient groups. Our findings do not aid in the discrimination between OLP and OLL and these conditions with or without contact allergic reactions.
\end{abstract}

Keywords: Oral lichen planus, Lichenoid lesions, Xerostomia, Salivary secretion, Total protein, slgA

\footnotetext{
* Correspondence: krrl@sund.ku.dk

${ }^{1}$ Section for Oral Pathology and Oral Medicine, Department of Odontology,

Faculty of Health and Medical Sciences, University of Copenhagen, 20 Noerre

Allé, DK-2200 Copenhagen N, Denmark

Full list of author information is available at the end of the article
} 


\section{Background}

Oral lichen planus (OLP) is one of the most common oral mucosal lesions affecting $0.5 \%$ to $2 \%$ of the adult population [1-4]. OLP mainly affects middle-aged and elderly, and is more prevalent in women than in men [5-7]. OLP may present as reticular, erythematous, ulcerative, plaque-like, bullous and papular lesions affecting predominantly the buccal mucosa, gingiva and tongue $[3,5,6,8]$.

The etiology is unknown, but the pathogenesis is believed to involve a T-cell-mediated response. However, the mechanisms triggering the T-cells to enter the oral epithelium and to accumulate in the superficial lamina propria as well the triggering mechanisms behind basal keratinocyte apoptosis are not fully understood, and may involve both antigen-specific and non-specific mechanisms [9]. The diagnosis of OLP is based on fulfillment of clinical and histopathological criteria $[10,11]$. Lesions that are clinically and histopathologically similar to OLP may occur as a reaction to certain systemic medications [12] or dental materials [13-15] and are referred to as oral lichenoid lesions (OLL). Also oral hygiene substances, like flavorings, may trigger lichenoid contact sensitivity reactions $[16,17]$. At present it may be difficult to distinguish between OLP and OLL. Patients with the erythematous and ulcerative type of OLP often suffer from severe oral mucosal soreness, including burning and itching sensations, particularly in relation to the intake of spicy and acidic food, which may have a negative impact on oral functions as well as the patients' quality of life and wellbeing [18]. In addition, we have previously shown that $45 \%$ of patients with erythematous and ulcerative OLP also suffer from xerostomia (the subjective feeling of dry mouth) and a sensation of very viscous saliva [18]. These findings have been substantiated by other studies, demonstrating an association between OLP and OLL and xerostomia $[19,20]$. OLP and OLL may occur in conditions that are associated with xerostomia and salivary gland hypofunction, including Sjögren's syndrome, hepatitis C infection, type 1 diabetes, and graft-versus-host disease [21-26]. Patients with these conditions often also display immune-mediated sialoadenitis which may affect the function of the salivary glands. However, it is still debatable whether the complaints of dry mouth and other sicca symptoms should be ascribed the OLP/OLL alone or whether they reflect a pathogenic association to systemic autoimmune diseases in which xerostomia and salivary gland dysfunction are common clinical manifestations. We have previously shown that only $15 \%$ of the $45 \%$ OLP patients who complained about xerostomia, actually had hyposalivation (i.e., an unstimulated whole saliva flow rate below $\leq 0.10 \mathrm{ml} / \mathrm{min}$ ), and this could be related to a daily intake of cardiovascular medications including antihypertensives known to cause xerostomia and/or hyposalivation $[18,27,28]$. A study on salivary gland function and morphology showed that $87 \%$ of 39 patients with OLP had a low or very low unstimulated whole saliva flow rate, whereas the chewing-stimulated whole saliva flow rate, the buffering capacity and the salivary $\mathrm{pH}$ level were normal. A labial salivary gland biopsy was performed in 15 patients, revealing lymphocytic infiltration (in 80\%), acinar atrophy (in 93\%), fibrosis, fatty degeneration, or ductal changes. However, although they displayed comparable signs of Sjögren's syndrome, none fulfilled the classification criteria [29]. Another study on 100 patients with OLP found no associations between OLP and low unstimulated and citric acid stimulated whole saliva flow rates [30].

Xerostomia denotes the subjective feeling of oral dryness, and it is often caused by salivary gland hypofunction, but may occur in the presence of an apparently normal salivary secretion, indicating that not only quantity but also quality of saliva is of importance to the feeling of oral comfort [31, 32]. Accordingly, changes in saliva composition may affect mucosal adhesiveness and lubrication [33]. Moreover, salivary secretion can be reduced as much as $50 \%$ of a person's normal whole saliva flow rate, before the sensation of oral dryness occurs [34]. Regarding changes in saliva composition, it has recently been reported that the total protein concentration was higher in patients with OLP than in healthy control subjects [35]. However, Gandara and co-workers found no differences between patients with OLP and healthy controls in terms of saliva flow rates, salivary proteins and electrolytes [36]. Moreover, it has been shown that patients with OLP and OLL have higher levels of salivary immunoglobulins IgA and IgG than healthy control subjects [35, 37, 38]. Secretory immunoglobin A (sIgA) and immunoglobulin G (IgG) are the two major classes of antibodies in human saliva comprising, 90-98\% and 1-10\%, respectively [39]. It has been suggested that both serum and salivary immunoglobulins, and particularly sIgA and IgG, play an important role in the pathogenesis of OLP, and it has also been hypothesised that the levels of sIgA and IgG could be useful in discriminating the OLP from OLL [38].

The aim of this cross-sectional study was to examine whether xerostomia, the degree of xerostomia, the unstimulated and chewing-stimulated whole saliva and citric-acid-stimulated parotid saliva flow rates, and the salivary concentration of total protein and sIgA could be used as diagnostic tools in discriminating between patients with OLP, OLL and generalised stomatitis with and without contact allergy.

We hypothesised that patients with OLP, OLL and generalised stomatitis and concomitant contact allergies have a higher frequency of xerostomia, more severe xerostomia, lower unstimulated and chewing-stimulated whole saliva and citric-acid-stimulated parotid saliva flow rates, and higher salivary concentration of total protein and sIgA than patients with OLP, OLL and stomatitis but without 
contact allergies; and healthy age- and gender-matched control subjects.

\section{Methods}

The study was approved by the Regional Ethics Committee, Copenhagen, Denmark (no. H-3-2013-033, March $26^{\text {th }} 2013$ ) and conducted according to the Declaration of Helsinki. The participants were both informed by letter and orally and all gave written consent prior to inclusion.

\section{Study participants}

One hundred and thirty-four consecutive patients referred to the Clinic for Oral Medicine, Department of Odontology, Faculty of Health and Medical Sciences, University of Copenhagen, due to symptoms and signs of oral mucosal diseases were screened for inclusion in the study. Fifty-two Caucasian patients were eligible for inclusion of whom 49 (94.2\%) completed the study. The remaining patients were excluded due to other diagnoses than OLP, OLL and stomatitis, and in cases where intake of medication was suspected as the eliciting cause of their mucosal lesions. Twenty-nine healthy age- and gender-matched subjects were included via the Danish website for study subjects (www.forsoegsperson.dk). The exclusion criteria for this group were past or current history of systemic and oral diseases as well as intake of medication. As it proved difficult to recruit healthy non-medicated control subjects at the age above 65 years, 4 persons taking antihypertensives (but otherwise healthy) were matched to the patients with regard to gender, age and type of antihypertensive agent.

An oral smear was taken from all study participants prior to inclusion in order to exclude oral candidiasis as it may mimic other mucosal lesions, e.g. erythematous OLP. A superimposed fungal infection may also masquerade the pattern of OLP lesions and be the cause of oral symptoms. The smear was stained with Periodic Acid Schiff and evaluated cytologically for presence of yeast hyphae and spores. Eleven patients, but none of the healthy subjects, had an oral candidiasis. They were treated with nystatin for 4 weeks, prior to inclusion. The treatment had no impact on their oral symptoms, but according to a repeated smear, the hyphae and spores were eliminated.

All patients had oral symptoms including stinging and burning sensations, and were diagnosed with OLP, OLL or generalised stomatitis. The patients with OLP and OLL were clinically characterised by various combinations of reticular, erythematous, ulcerative and plaquelike mucosal changes and the diagnosis was confirmed by a biopsy and histopathological examination according to van der Meij and van der Waal [11]. However, this method to discriminate between OLP and OLL still needs to be validated in larger scale studies. The patients diagnosed with stomatitis were characterised by having a more diffuse, widespread oral mucosal erythema. The patients with OLP and OLL were pooled in one group as a strict distinction between the two entities was difficult to make.

All participants underwent a mucosal biopsy at the Department of Odontology, University of Copenhagen, and were referred for patch testing for contact allergy at the Department of Dermatology and Allergy, Gentofte University Hospital. Patch testing to the European baseline series, a toothpaste series and a dental material series were done according to the European Society of Contact Dermatitis (ESCD) guidelines [40]. Moreover, all participants had a check of the serum levels of thyroid stimulating hormone (TSH). They also underwent an interview including standardised questions regarding present and past systemic diseases, including allergies, daily intake of medication, habits regarding alcohol consumption, tobacco smoking and oral hygiene. Data on smoking habits was used to categorize participants as never smokers, former smokers and current smokers. Data on alcohol consumption was used to pool the participants in groups of never consuming alcohol, occasionally or daily consumption of alcohol.

\section{Assessment of xerostomia}

Apart from being questioned about symptoms of the oral mucosa like stinging, burning and roughness, the participants were also asked whether they had taste disturbances and the character of their disturbances in taste perception. Moreover, the participants were asked whether they had symptoms of dry mouth, and if present they were also asked to score the severity of xerostomia using a categorized questionnaire based on Beck's inventory scale on how questionnaires should be designed in order to obtain valid responses [41] including four degrees of severity (scores 0-3) [42]. The assessment also included potential affection of oropharyngeal functions and diurnal variation in the sensation of oral dryness (Table 1).

\section{Measurements of whole saliva and parotid saliva flow rates} One examiner (KRL) conducted the collection of the whole saliva and the parotid saliva. In brief, the participants were asked to refrain from drinking, eating, chewing chewing-gum/pastilles, tooth brushing and smoking for one hour prior to the salivary measurement. The whole saliva samples were collected using the draining method [43]. Unstimulated and paraffin-chewingstimulated whole saliva flow rates (UWS and SWS, respectively) were sampled over a 15 -min and a $5 \mathrm{~min}$ period, respectively. The citric acid stimulated parotid saliva (SPS) was sampled over a 5-min period [43]. To minimize the influence of the circadian cycle on the composition and flow rate of saliva, all procedures were performed in the same order between $9.00 \mathrm{am}$ and 
Table 1 Questionnaire regarding xerostomia and scoring of the severity of xerostomia by patients and healthy controls [41]

\begin{tabular}{lll}
\hline Category & $\begin{array}{l}\text { Patients } \\
n=49\end{array}$ & $\begin{array}{l}\text { Healthy } \\
\text { controls } n=29\end{array}$ \\
\hline No feeling of dry mouth (score 0) & $53.1 \%$ & $79.3 \%$ \\
Slight feeling of dry mouth (score 1) & $26.5 \%$ & $20.7 \%$ \\
Severe feeling of dry mouth (score 2) & $8.2 \%$ & $0 \%$ \\
Annoying feeling of dry mouth & $12.2 \%$ & $0 \%$ \\
making speech difficult (score 3) & & \\
Difficulty swallowing due to xerostomia & $12.2 \%$ & $0 \%$ \\
Difficulty chewing due to xerostomia & $10.2 \%$ & $0 \%$ \\
Difficulty in eating dry food substances & $32.7 \%$ & $0 \%$ \\
due to xerostomia & & \\
Taste perception different from normal & $28.6 \%$ & $0 \%$ \\
-increased taste perception & $4.1 \%$ & $0 \%$ \\
-decreased taste perception & $18.4 \%$ & $0 \%$ \\
-no taste perception & $4.1 \%$ & $0 \%$ \\
-altered taste perception (dysgeusia) & $14.3 \%$ & $0 \%$ \\
$\quad$ Nocturnal xerostomia & $24.5 \%$ & $13.8 \%{ }^{\mathrm{a}}$ \\
$\quad$ Morning xerostomia & $36.7 \%$ & $24.1 \%{ }^{\mathrm{a}}$ \\
$\quad$ Daytime xerostomia & $22.4 \%$ & $3.4 \%$ \\
$\quad$ Evening xerostomia & $10.2 \%$ & $0 \%$ \\
$\quad$ Xerostomia at all times of the day & $10.2 \%$ & $0 \%$ \\
$\quad$ Waking up due to xerostomia & $34.7 \%$ & $17.2 \%{ }^{\mathrm{a}}$ \\
$\quad$ Duration of xerostomia, years & 2,88 & 2 \\
\hline
\end{tabular}

Number of participants reporting symptoms are given in percentage. ${ }^{\mathrm{a}}$ Reported to be caused by periodic snoring, in periods without snoring they did not have xerostomia

11.00 am every time. The saliva samples were immediately weighed so that the flow rates could be established and then portioned in $1.5 \mathrm{ml}$ Eppendorf tubes, centrifuged at $10.000 \mathrm{~g}$ for $20 \mathrm{~min}$ at $4^{\circ} \mathrm{C}$, portioned and frozen at $-80^{\circ}$ $C$ until further analysis. UWS values of $\leq 0.10 \mathrm{ml} / \mathrm{min}$ and SWS values of $\leq 0.70 \mathrm{ml} / \mathrm{min}$ were designated hyposalivation [42]. Patients with UWS flow rates below $0.20 \mathrm{ml} /$ min were designated as low secretors [44].

\section{Clinical oral examination}

One examiner (KRL) conducted the oral clinical examination, calibrated against an experienced clinical examiner (AMLP). The localisation, size and colour of the oral lesions were registered and clinical photos were taken. Also the clinical signs of mucosal dryness were registered.

\section{Analyses of salivary total protein and slgA}

The total protein concentration $(\mu \mathrm{g} / \mathrm{ml})$ in unstimulated and chewing-stimulated whole saliva was determined using a colorimetric assay [45]. An indirect enzyme immunoassay kit was used to determine SIgA in the whole saliva samples according to the protocol supplied by Salimetrics ${ }^{\oplus}$ (Salimetrics, PA, USA). The out-put of IgA $(\mu \mathrm{g} / \mathrm{min})$ was determined by each concentration was multiplied with the respective salivary flow rate $(\mathrm{ml} /$ $\min )$.

\section{Statistics}

The statistical analyses were performed using SPSS Version 22 (IBM). Fisher's test was used for analysis of distributions between the patient group, including those with or without a concomitant contact allergy, and the healthy control group. As the saliva flow rates (UWS, SWS and SPS) were not normally distributed in the patient group, the Mann-Whitney $U$ test was used. A $t$-test was used after fulfilling the Shapiro-Wilk's and Levene's tests, when comparing the data from the patients with OLL with and without a concomitant contact allergy. The Mann-Whitney $U$ test was used for comparisons in salivary total protein and sIgA between the patients and the healthy control subjects, and between patients with OLL with and without a concomitant contact allergy. Associations between variables were analysed by the Spearman rank order correlation test. Statistical significance was defined as $p<0.05$.

\section{Results}

About $85 \%$ of the participants were women, and the average age was 61 years (median 65 years, range 32-77 years). Three patients used tobacco on a daily basis and on average they had smoked 21.5 smoking pack years. Four healthy controls used tobacco daily and they had on average smoked 17.3 smoking pack years. Nineteen patients and 14 healthy controls had ceased smoking more than one year prior to the inclusion in the project. Seven patients and eleven healthy controls reported a daily consumption of alcohol. One male patient reported an alcohol consumption that exceeded the 21 units of alcohol per week limit as recommended by the Danish Health Authority. Forty-six (85.7\%) patients reported having one or more medical condition/disease (median 2, range 1-12). The most common ones included recurrent herpes labialis (herpes simplex virus, $n=20)$, hypertension $(n=11)$, osteoarthritis $(n=12)$, contact dermatitis $(n=9)$ and asthma $(n$ $=7$ ). Furthermore, 6 patients had pollen allergy, 7 had contact allergy to nickel and 5 had allergy to fragrance ingredients. These were all confirmed prior to the inclusion in the project by testing. General information on the participants can be seen in Table 2 .

Thirty-two (65.3\%) patients reported daily intake of prescribed medication (median 2, range 1-10), most commonly antihypertensives. Sixteen (32.6\%) patients had a daily intake of more than two different types of medication (i.e., polypharmacy), 7 patients took two types of medication daily and 9 patients one type of medication daily. Three female patients (6\%) had hypothyroidism and one female patient had hyperthyroidism. Blood samples 
Table 2 Clinical characteristics of the patients and healthy controls

\begin{tabular}{lll}
\hline & $\begin{array}{c}\text { Patients } \\
(n=49)\end{array}$ & $\begin{array}{l}\text { Healthy controls } \\
(n=29)\end{array}$ \\
\hline Female:male ratio & $42: 7$ & 25.4 \\
Age, years & $\begin{array}{l}61.0 \pm 10.3 \\
(\text { range } 31-77)\end{array}$ & $\begin{array}{l}58.2 \pm 12.1 \\
\text { (range 23-73) }\end{array}$ \\
Smoking, percentage (smoking pack year) & \\
-non & $55.1 \%(0)$ & $37.9 \%(0)$ \\
-former & $38.8 \%(21.2)$ & $48.3 \%(12.8)$ \\
-current & $6.1 \%(21.5)$ & $13.8 \%(17.3)$ \\
Alcohol consumption, percentage & \\
-never & $61.2 \%$ & $17.2 \%$ \\
-occasionally & $24.5 \%$ & $44.8 \%$ \\
-daily & $14.3 \%$ & $37.9 \%$ \\
Chronic medical conditions (no. given in\%) & \\
-herpes labialis & $40.8 \%$ & $41.4 \%$ \\
-hypertension & $24.5 \%$ & $6.9 \%$ \\
-osteoarthritis & $24.5 \%$ & $6.9 \%$ \\
-hypercholesterolaemia & $10.2 \%$ & $6.9 \%$ \\
-allergic rhinitis & $12.2 \%$ & $0 \%$ \\
-asthma & $14.3 \%$ & $3.4 \%$ \\
-contact allergy & $38.8 \%$ & $34.5 \%$ \\
\hline
\end{tabular}

Data are given in mean and SD

analyzed for levels of thyroid stimulating hormone (TSH) revealed that all patients but two had TSH levels within the normal range. In one patient with known hypothyroidism, the level of TSH was elevated, and her medical treatment was consequently regulated. In another patient with known hyperthyroidism, the level of TSH was decreased, and her medical treatment was adjusted accordingly. In none of these two patients, the adjustments in their treatment lead to changes in their oral condition. Three healthy control subjects showed elevated levels of TSH and were referred to additional medical examinations of possible hypothyroidism. There were no associations between age, gender and the number of medical conditions/diseases and the number of medication taken on daily basis.

The diagnosis of OLP was established in 19 patients and additionally 19 patients were diagnosed with OLL. Fifteen patients had the erosive form of OLP at the time of the examination. As an exact distinction between OLP and OLL was difficult to make, we pooled the patients in one group comprising both OLP and OLL. None of the patients were diagnosed with hepatitis C, Sjögren's syndrome or any other autoimmune disease. The diagnosis of stomatitis was made in 11 patients (22.4\%). Nineteen patients (38.8\%) and 10 (34.5\%) healthy control subjects were diagnosed with contact allergy, primarily to fragrance mix. Contact allergy to aroma substances in oral hygiene products differed significantly between the patients and the healthy control subjects $(p=0.02)$, primarily in patients with OLP and OLL $(p=0.01)$. Spearmint was the most common allergen in the patient group, whereas it was cassia oil in the healthy controls. The substances that the participants primarily displayed positive patch test reaction to are listed in Table 3.

Table 1 shows the distribution of xerostomia in terms of severity of xerostomia and any diurnal variations in the patient group with OLP/OLL and stomatitis and the healthy control group. Expectedly, the patients had significantly more complaints of xerostomia and more severe xerostomia than the healthy control subjects $(p<$ 0.001). However, there were no differences in the frequency of xerostomia between patients with OLP, OLL and stomatitis, and patients with and without a concomitant contact allergy $(p=0.30)$. There were no associations between the presence and severity of xerostomia and age, gender, number of medical conditions/ diseases, including allergies, or the number of medications taken on a daily basis. More female patients than male patients reported xerostomia, but the majority of the study population also comprised women. $28.6 \%$ of the patients reported that they experienced their taste perception to be different from normal, primarily as a decreased or an altered taste perception.

The salivary flow rates are listed in Table 4 . No differences could be found between the patient group and the healthy control group or between the patients with OLP,

Table 3 Substances that the participants primarily displayed positive patch test reaction to

\begin{tabular}{lll}
\hline Substances with positive patch test reactions & $\begin{array}{l}\text { Patients } \\
(n=49)\end{array}$ & $\begin{array}{l}\text { Healthy controls } \\
(n=29)\end{array}$ \\
\hline Perfume mix & 7 & 4 \\
Balsam of Peru & 5 & 1 \\
Colophony & 4 & 0 \\
Spearmint & 4 & 0 \\
Cassai oil & 3 & 1 \\
Carvone & 2 & 0 \\
Cinnemaldehyd & 1 & 0 \\
Nickel & 2 & 4 \\
Mercury & 2 & 0 \\
2-hydroxyethyl-methacrylate & 2 & 0 \\
Methylmethacrylate (MMA) & 1 & 0 \\
Ethylen glycol dimethacrylate (EDGMA) & 1 & 0 \\
Palladium & 1 & 1 \\
Cobalt & 1 & 1
\end{tabular}

Some participants displayed more than one positive patch test reaction which explains why the total number adds up to more than the number of patients and healthy controls [56] 
Table 4 Unstimulated (UWS), paraffin-chewing-stimulated (SWS) and citric-acid-stimulated parotid (SPS) saliva flow rates ( $\mathrm{ml} / \mathrm{min}$ ), total salivary protein concentration $(\mu \mathrm{g} / \mathrm{min})$ and salivary slgA levels $(\mu \mathrm{g} / \mathrm{min})$ in unstimulated and chewing-stimulated whole saliva

\begin{tabular}{llll}
\hline & $\begin{array}{l}\text { Patients } \\
(n=49)\end{array}$ & $\begin{array}{l}\text { Healthy controls } \\
(n=29)\end{array}$ & $p$-value \\
\hline UWS (ml/min) & $0.33 \pm 0.21$ & $0.39 \pm 0.25$ & 0.33 \\
SWS (ml/min) & $1.69 \pm 1.03$ & $1.75 \pm 0.72$ & 0.25 \\
SPS (m//min) & $0.15 \pm 0.16$ & $0.15 \pm 0.12$ & 0.56 \\
$\begin{array}{l}\text { Total protein concentration } \\
\text { ( } \mu \mathrm{g} / \mathrm{min})\end{array}$ & & & \\
$\quad$-UWS & $1047.9 \pm 633.0$ & $1131.0 \pm 640.1$ & 0.86 \\
$\quad$-SWS & $4317.6 \pm 2414.6$ & $4090.0 \pm 1689.1$ & 0.58 \\
Levels of slgA ( $\mu \mathrm{g} / \mathrm{min})$ & & & \\
$\quad$-UWS & $48.6 \pm 29.5$ & $47.8 \pm 21.79$ & 0.82 \\
$\quad$-SWS & $96.1 \pm 51.7$ & $82.4 \pm 38.1$ & 0.19 \\
\hline
\end{tabular}

All data are given in mean and SD

OLL and generalised stomatitis with and without a concomitant contact allergy regarding the UWS, SWS and SPS flow rates. However, the UWS flow rates tended to be lower in patients with OLP/OLL and concomitant contact allergy than in those without contact allergy $(p=0.05)$. One patient had severely reduced UWS, SWS and SPS with UWS flow rates below $0.10 \mathrm{ml} / \mathrm{min}$ and SWS flow rates $\leq 0.70 \mathrm{ml} / \mathrm{min}$. Three other patients had UWS hyposalivation, whereas 10 patients were characterised as low secretors. Moreover, two patients had SWS flow rates $\leq 0.70 \mathrm{ml} / \mathrm{min}$, but normal UWS flow rates. One healthy control subject had UWS hyposalivation and 8 were low secretors, but SWS flow rates were in the normal range. There were no associations between the salivary flow rates (UWS, SWS and SPS) and age, gender, number of medical conditions/diseases, the number of medications taken on a daily basis or the prevalence and severity of xerostomia. The patients with UWS hyposalivation had a salivary gland biopsy taken from the minor glands in the mucosa of the lower lip under the suspicion of Sjögren's syndrome. None of them had focal lymphocytic infiltration in their glandular tissue or serum autoantibodies, excluding Sjögren's syndrome. The SPS flow rates did not differ between the patients and the healthy controls or between the patients with and without a concomitant contact allergy.

The total protein concentration in terms of output $(\mu \mathrm{g} / \mathrm{min})$ in unstimulated and chewing-stimulated whole saliva from patients and healthy control subjects are listed in Table 4. The patients had higher total protein concentrations in unstimulated whole saliva while it was lower in chewing-stimulated whole saliva but the differences were not significant ( $p=$ 0.58 and $p=0.86$ respectively), whereas there were no differences in the total salivary protein concentrations when comparing patients with OLL with and without a concomitant contact allergy.

The levels of sIgA in terms of output $(\mu \mathrm{g} / \mathrm{min})$ in both unstimulated and chewing-stimulated whole saliva from patients and healthy control subjects are shown in Table 4. The levels of sIgA were higher in both unstimulated and chewing-stimulated whole saliva in patients than in healthy control subjects but the difference was not significant $(p=0.82$ and $p=0.19$ respectively). No difference could be found in the salivary sIgA levels when comparing patients with OLP/OLL with and without a concomitant contact allergy.

\section{Discussion}

The aim of this cross-sectional study was to determine if xerostomia, degree of xerostomia, UWS, SWS and SPS flow rates and levels of salivary protein concentration and sIgA could be used in discriminating between patients with OLP/OLL and generalised stomatitis with or without concomitant allergy and healthy control subjects.

The majority of patients included in this study were females, which reflects the fact that more women than men are referred to medical clinics and that OLP/OLL are more prevalent in women. However, it also explains the high prevalence xerostomia and other symptoms, the high number of medical diseases/medical conditions and number of medications taken on a daily basis in our study. Furthermore, the age of onset of OLP also indicate that gender hormones may be involved in the pathogenesis via immunological and endocrinological changes affecting the oral mucosa and the immunological response and making the mucosa more susceptible to oral diseases like OLP and allergic reactions.

The diagnosis of OLP and OLL was established in 38 patients (77.6\%) according to the recommendations of van der Meij and van der Waal [11]. Although the latter seeks to differentiate between OLP and OLL based on clinicopathological criteria, the value of distinction between them is still controversial. The remaining 11 patients were diagnosed with generalised stomatitis. These patients displayed more diffuse reactions varying from barely visible erythema to bright red erythema anywhere in the oral mucosa. Erosions and hyperkeratosis were also seen as previously described [46]. In our study, we did not find a clear distinction between OLP and OLL with respect to the symptoms and the clinicopathological features.

It is well known that OLL may occur as adverse reactions to systemic drugs including angiotensin-converting enzyme (ACE) inhibitors and beta-blockers [12, 47]. In this study, we eliminated cases in which there was a clear temporal association between the intake of medication and the onset of oral symptoms and signs of OLL. In these cases we found that discontinuation of the 
medication also resulted in a gradual disappearance of the lichenoid reactions.

As expected, xerostomia was reported significantly more often by the patients (46.9\%) than healthy control subjects (20.7\%). We did not find any differences between the patients with OLP/OLL and generalised stomatitis with and without contact allergies in terms of prevalence and severity of xerostomia. It is well-known that certain systemic diseases and the intake of certain medications as well as the number of diseases and medications are associated with xerostomia $[28,48-51]$. In our study, $65.3 \%$ of the patients reported daily intake of medication and $32.6 \%$ had an intake of more than 2 different agents on a daily basis, which is reflecting the medication intake in the background population. Several of the medications taken by the participants in our study are known to be "xerogenic" $[27,28]$. This might explain the difference in the reports of xerostomia between the patients and the healthy controls. However, we did not find significant associations between the number of medical conditions/diseases, the number of medications and the presence and severity of xerostomia, which may reflect the fact that there were relatively few observations in each category of diseases and medications. However, there was tendency towards an association between the intake of antihypertensives and xerostomia. On the other hand, the sensation of dry mouth could also be solely related to OLP or OLL as previously described [18-20]. It is also well-known that women report xerostomia more frequently than men [48] and, as mentioned earlier, the majority of patients included in this study were women. In the healthy control group, 20.7\% reported a "slight sensation of dry mouth", which was related to awakening and "snoring". Xerostomia is often associated with salivary gland hypofunction, but in our study we did not find any associations between xerostomia, the severity of xerostomia and the salivary flow rates. Thus, it is likely that the inflammatory changes in the oral mucosa due to OLP, OLL and generalised stomatitis lead the sensory disturbances, including sensation of oral dryness [52].

We found no significant differences in UWS, SWS and SPS flow rates between the patients with and without concomitant allergies and the healthy control subjects, although there was a tendency towards lower UWS flow rates in patients with OLP/OLL and a concomitant contact allergy. Our findings are in concordance with several other studies showing that the salivary flow rates in patients diagnosed with OLP do not differ from the salivary flow rates in healthy control subjects [18, 20, 36, 37].

We found no associations between age, gender and the number of diseases and the number of medication taken on daily basis, which can be ascribed to the very narrow variation in age (the median age was 65 years).
We found higher total protein concentrations in chewing-stimulated saliva samples from patients with OLP/OLL and generalised stomatitis compared to those from healthy control subjects whereas the opposite could be seen in the unstimulated saliva samples. These differences were not significant. Changes in saliva composition can contribute to explain sensation of dry mouth. Our findings are in concordance with Gandara et al. [36] and Artico et al. [20], who found no differences in total protein concentration between patients with OLP and healthy controls. We found no difference either in the total salivary protein concentration between patients with OLP/ OLL with and without a concomitant contact allergy.

In our study, the levels of salivary sIgA were higher in both unstimulated and chewing-stimulated whole saliva samples from patients than in those from the healthy control subjects, but the difference was not significant. Previous findings on the levels of salivary sIgA concentrations are contradictory [35-38]. SIgA is an important part of the immune defense of mucosal surfaces. Salivary IgA can be used to evaluate the immune state and very high levels can indicate underlying pathology [37, 53, 54]. We found no difference in the salivary sIgA levels when comparing patients with OLP/OLL with and without a concomitant contact allergy.

A recent study showed that thyroid disease was present in 33 of 215 (15.3\%) of patients with OLP compared to 12 of 215 (5.2\%) in a control group [55]. However, in our study only 4 patients $(6.1 \%)$ had a thyroid disease and the serum levels of TSH revealed that three of the healthy control might have hypothyroidism.

It is likely that the higher prevalence and more severe xerostomia, the higher salivary concentrations of total protein and levels of $\operatorname{sg} \mathrm{A}$ that we find may be ascribed to a higher degree of anxiety, depression and sleep disturbances in the patient group. These speculations are supported by a recent study of Lopez-Jornet et al. [35] showing that patients OLP presented worse psychological profiles and sleep disturbances, and also higher values for sIgA, cortisol, and total proteins than control subjects.

\section{Conclusion}

Our findings indicate that OLP, OLL and generalised stomatitis are associated with xerostomia. However, xerostomia was not associated with reduction in the salivary flow rates, the number of diseases or number of medications taken on a daily basis, age or gender. There were no differences in UWS, SWS and SPS flow rates between the patients with OLP/OLL and generalised stomatitis and healthy control subjects. On the other hand, the salivary concentrations of total protein and the levels of sIgA were higher in the patient group than in the healthy control group even though the difference was not significant. However, these findings 
do not aid in the discrimination between OLP and OLL and these conditions with or without contact allergic reactions.

\section{Abbreviations}

ESCD: European Society of Contact Dermatitis; IgG: Immunoglobulin G; OLL: Ora lichenoid lesions; OLP: Oral lichen planus; slgA: Secretory immunoglobulin A: SPS: Citric acid stimulated parotid saliva; SWS: Chewing stimulated whole saliva; TSH: Thyroid stimulating hormone; UWS: Unstimulated whole saliva

\section{Acknowledgements}

Thanks to laboratory technicians Louise Rosgaard Duus and Joan Lykkeaa for assistance with the laboratory work.

\section{Funding}

Faculty of Medical and Health Science, University of Copenhagen, the Danish Dental Association Research Foundation and Toyota Fonden, Denmark.

\section{Availability of data and materials}

The clinical data and personal details will not be made available in order to protect the participants' identity.

\section{Authors' contributions}

Substantial contributions to the design of the study: AMLP, JDJ and JR. Substantial contributions to the acquisition of data: KRL, AMLP, JDJ, JR and CZ. Substantial contributions to the analysis of data: AMLP and KR. Substantial contributions to the interpretation of data: KRL and AMLP. Authoring the first draft: KRL. Critically revising the manuscript: All authors. All authors read and approved the final manuscript.

\section{Competing interests}

The authors declare that they have no competing interests.

\section{Consent for publication}

Not applicable.

\section{Ethics approval and consent to participate}

The study was approved by the Regional Ethics Committee, Copenhagen, Denmark (no. H-3-2013-033, March 26th 2013) and conducted according to the Declaration of Helsinki. The participants were both informed by letter and orally and all gave written consent prior to inclusion.

\section{Publisher's Note}

Springer Nature remains neutral with regard to jurisdictional claims in published maps and institutional affiliations.

\section{Author details \\ ${ }^{1}$ Section for Oral Pathology and Oral Medicine, Department of Odontology, Faculty of Health and Medical Sciences, University of Copenhagen, 20 Noerre Allé, DK-2200 Copenhagen N, Denmark. ${ }^{2}$ National Allergy Research Centre, Department of Dermatology and Allergy, Gentofte University Hospital, 28 Kildegaardsvej, DK-2900 Hellerup, Denmark. ${ }^{3}$ Department of Dermatology and Allergy, Gentofte University Hospital, 28 Kildegaardsvej, DK-2900 Hellerup, Denmark. ${ }^{4}$ Section for Community Dentistry, Department of Odontology, Faculty of Health and Medical Sciences, University of Copenhagen, 20 Noerre Allé, DK-2200 Copenhagen N, Denmark.}

Received: 10 February 2017 Accepted: 16 June 2017

Published online: 29 June 2017

\section{References}

1. Axéll T, Rundquist L. Oral lichen planus-a demographic study. Community Dent Oral Epidemiol. 1987;15:52-6.

2. Bowers KE, Sexton J, Sugerman PB. Commentary. Clin Dermatol. 2000;18:497-8.

3. McCartan BE, Healy CM. The reported prevalence of oral lichen planus: a review and critique. J Oral Pathol Med. 2008:37:447-53.

4. Pinto A, Khalaf M, Miller CS. The practice of oral medicine in the United States in the twenty-first century: an update. Oral Surg Oral Med Oral Pathol Oral Radiol. 2015;119:408-15.
5. Silverman Jr S, Gorsky M, Lozada-Nur F, Giannotti K. A prospective study of findings and management in 214 patients with oral lichen planus. Oral Surg Oral Med Oral Pathol. 1991;72:665-70.

6. Scully C, Beyli M, Ferreiro MC, Ficarra G, Gill Y, Griffiths M, Holmstrup P, Mutlu S, Porter S, Wray D. Update on oral lichen planus: etiopathogenesis and management. Crit Rev Oral Biol Med. 1998:9:86-122.

7. Kovac-Kovacic M, Skaleric U. The prevalence of oral mucosal lesions in a population in Ljubljana, Slovenia. J Oral Pathol Med. 2000;29:331-5.

8. Thorn JJ, Holmstrup P, Rindum J, Pindborg JJ. Course of various clinical forms of oral lichen planus. A prospective follow-up study of 611 patients. J Oral Pathol. 1988;17:213-8.

9. Sugerman PB, Savage NW, Walsh $\sqcup$, Zhao ZZ, Zhou XJ, Khan A, Seymour GJ, Bigby M. The pathogenesis of oral lichen planus. Crit Rev Oral Biol Med. 2002:13:350-65.

10. Kramer IRH, Lucas RB, Pindborg JJ, Sobin LH. Definition of leukoplakia and related lesions: An aid to studies on oral cancer. Oral Surg Oral Med Oral Pathol. 1978;46:518-39.

11. van der Meij EH, van der Waal I. Lack of clinicopathologic correlation in the diagnosis of oral lichen planus based on the presently available diagnostic criteria and suggestions for modifications. J Oral Pathol Med. 2003;32:507-12.

12. Porter SR, Scully C. Adverse drug reactions in the mouth. Clin Dermatol. 2000;18:525-32.

13. Lind PO. Oral lichenoid reactions related to composite restorations. Preliminary Report Acta Odontol Scand. 1988;46:63-5.

14. Bolewska J, Holmstrup P, Møller-Madsen B, Kenrad B, Danscher G. Amalgam associated mercury accumulations in normal oral mucosa, oral mucosal lesions of lichen planus and contact lesions associated with amalgam. J Oral Pathol Med. 1990;19:39-42.

15. Scully C, Carrozzo M. Oral mucosal disease: Lichen planus. Br J Oral Maxillofac Surg. 2008;46:15-21.

16. Miller RL, Gould AR, Bernstein ML. Cinnamon-induced stomatitis venenata, Clinical and characteristic histopathologic features. Oral Surg Oral Med Oral Pathol. 1992:73:708-16.

17. Yiannias JA, el-Azhary RA, Hand JH, Pakzad SY, 3rd Rogers RS. Relevant contact sensitivities in patients with the diagnosis of oral lichen planus. J Am Acad Dermatol. 2000;42:177-82.

18. Pedersen AM, Andersen TL, Reibel J, Holmstrup P, Nauntofte B. Oral findings in patients with primary Sjögren's syndrome and oral lichen planus-a preliminary study on the effects of bovine colostrum-containing oral hygiene products. Clin Oral Investig. 2002;6:11-20.

19. Colquhoun AN, Ferguson MM. An association between oral lichen planus and a persistently dry mouth. Oral Surg Oral Med Oral Pathol Oral Radiol Endod. 2004;98:60-8.

20. Artico G, Freitas RS, Santos Filho AM, Benard G, Romiti R, Migliari DA. Prevalence of Candida spp., xerostomia, and hyposalivation in oral lichen planus-a controlled study. Oral Dis. 2014;20:e36-41.

21. Bermejo Fenoll A, López Jornet MP. Oral lichen planus and Sjogren's syndrome. 2 cases of association. Av Odontoestomatol. 1991;7:29-33. 36, 38

22. Petrou-Amerikanou C, Markopoulos AK, Belazi M, Karamitsos D, Papanayotou P. Prevalence of oral lichen planus in diabetes mellitus according to the type of diabetes. Oral Dis. 1998;4:37-40.

23. Sato M, Tokuda N, Fukumoto T, Mano T, Sato T, Ueyama $Y$ Immunohistopathological study of the oral lichenoid lesions of chronic GVHD. J Oral Pathol Med. 2006:35:33-6.

24. Lodi G, Pellicano R, Carrozzo M. Hepatitis C virus infection and lichen planus: a systematic review with meta-analysis. Oral Dis. 2010;16:601-12.

25. Hull K, Kerridge I, Avery S, McCullough M, Ritchie D, Szer J. Oral chronic graft-versus-host disease in Australia: clinical features and challenges in management. Intern Med J. 2015;45:702-10.

26. Alaizari NA, Al-Maweri SA, Al-Shamiri HM, Tarakji B, Shugaa-Addin B. Hepatitis C virus infections in oral lichen planus: a systematic review and meta-analysis. Aust Dent J. 2016;61:282-7.

27. Smidt D, Torpet LA, Nauntofte B, Heegaard KM, Pedersen AML. Associations between oral and ocular dryness, labial and whole salivary flow rates, systemic diseases and medications in a sample of older people. Community Dent Oral Epidemiol. 2011;39:276-88

28. Villa A, Wolff A, Narayana N, Dawes C, Aframian DJ, Lynge Pedersen AM, Vissink A, Aliko A, Sia YW, Joshi RK, McGowan R, Jensen SB, Kerr AR, Ekström J. Proctor G. World Workshop on Oral Medicine Vl: a systematic review of medication-induced salivary gland dysfunction. Oral Dis. 2016;22:365-82.

29. Lundström IM, Anneroth KG, Bergstedt HF Salivary gland function and changes in patients with oral lichen planus. Scand J Dent Res. 1982;90:443-58. 
30. Ramon C, Bagan JV, Milian MA, Jimenez Y, Lloria E. Quantitative analysis of saliva in patients with oral lichen planus: a study of 100 cases. Med Oral. 2000;5:187-92.

31. Tabak LA, Levine MJ, Mandel ID, Ellison SA. Role of salivary mucins in the protection of the oral cavity. J Oral Pathol. 1982;11:1-17.

32. Fox PC, Busch KA, Baum BJ. Subjective reports of xerostomia and objective measures of salivary gland performance. J Am Dent Assoc. 1987:115:581-4.

33. Dawes C, Pedersen AM, Villa A, Ekström J, Proctor GB, Vissink A, Aframian D, McGowan R, Aliko A, Narayana N, Sia YW, Joshi RK, Jensen SB, Kerr AR, Wolff A. The functions of human saliva: A review sponsored by the World Workshop on Oral Medicine VI. Arch Oral Biol. 2015;60:863-74.

34. Dawes C. Physiological factors affecting salivary flow rate, oral sugar clearance, and the sensation of dry mouth in man. J Dent Res. 1987;66:648-53.

35. Lopez-Jornet P, Cayuela CA, Tvarijonaviciute A, Parra-Perez F, Escribano D, Ceron J. Oral lichen planus: saliva biomarkers cortisol, immunoglobulin A, adiponectin. J Oral Pathol Med. 2016;45:211-7.

36. Gandara BK, Izutsu KT, Truelove EL, Mandel ID, Sommers EE, Ensign WY. Sialochemistry of whole, parotid, and labial minor gland saliva in patients with oral lichen planus. J Dent Res. 1987;66:1619-22.

37. Sistig S, Vucićević-Boras V, Lukac J, Kusić Z. Salivary lgA and lgG subclasses in oral mucosal diseases. Oral Dis. 2002;8:282-6.

38. Ghaleyani P, Sardari F, Akbari M. Salivary lgA and lgG in oral lichen planus and oral lichenoid reactions diseases. Adv Biomed Res. 2012;1:73.

39. Brandtzaeg P. Secretory immunity with special reference to the oral cavity. J Oral Microbiol. 2013;5. doi:10.3402/jom.v5i0.20401.

40. Johansen JD, Aalto-Korte K, Agner T, Andersen KE, Bircher A, Bruze M, Cannavó A, Giménez-Arnau A, Gonçalo M, Goossens A, John SM, Lidén C, Lindberg M, Mahler V, Matura M, Rustemeyer T, Serup J, Spiewak R, Thyssen JP, Vigan M, White IR, Wilkinson M, Uter W. European Society of Contact Dermatitis guideline for diagnostic patch testing - recommendations on best practice. Contact Dermatitis. 2015;73:195-221.

41. Beck AT, Ward CH, Mendelson M, Mock J, Erbaugh J. An inventory for measuring depression. Arch Gen Psychiatry. 1961:4:561-71.

42. Pedersen AM, Reibel J, Nauntofte B. Primary Sjögren's syndrome (pSS): subjective symptoms and salivary findings. J Oral Pathol Med. 1999;28:303-11.

43. Pedersen AM, Reibel J, Nordgarden H, Bergem HO, Jensen JL, Nauntofte B. Primary Sjögren's syndrome: salivary gland function and clinical oral findings. Oral Dis. 1999;5:128-318.

44. Ericsson $Y$, Hardwick L. Individual diagnosis, prognosis and counselling for caries prevention. Caries Res. 1978;12 Suppl 1:94-102.

45. Pedersen AM, Bardow A, Nauntofte B. Salivary changes and dental caries as potential oral markers of autoimmune salivary gland dysfunction in primary Sjogren's syndrome. BMC Clin Pathol. 2005;5:4. doi:10.1186/1472-6890-5-4.

46. Isaac-Renton M, Li MK, Parsons LM. Cinnamon spice and everything not nice: many features of intraoral allergy to cinnamic aldehyde. Dermatitis. 2015;26:116-21.

47. Sugerman PB, Savage NW, Zhou X, Walsh LJ, Bigby M. Oral lichen planus. Clin Dermatol. 2000;18:533-9.

48. Nederfors T, Isaksson R, Mörnstad H, Dahlöf C. Prevalence of perceived symptoms of dry mouth in an adult Swedish population-relation to age, sex and pharmacotherapy. Community Dent Oral Epidemiol. 1997:25:211-6.

49. Sreebny LM, Schwartz SS. A reference guide to drugs and dry mouth-2nd edition. Gerodontology. 1997;14:33-47.

50. Pedersen AM, Bardow A, Jensen SB, Nauntofte B. Saliva and gastrointestinal functions of taste, mastication, swallowing and digestion. Oral Dis. 2002:8:117-29.

51. Pedersen AML. Diseases Causing Oral Dryness. In: Carpenter G, editor. Dry mouth - a clinical guide on causes, effects and treatments. Heidelberg: Springer; 2015. p. 7-31.

52. Niissalo S, Hampf G, Hietanen J, Malmström M, Solovieva S, Pertovaara A, Konttinen YT. Thermal sensation and pain in oral lichen planus and lichenoid reaction. J Oral Pathol Med. 2003;32:41-5.

53. Biocina-Lukenda D, Cekić-Arambasin A, Markeljević J, Buković D. Serum immunoglobulins $\lg G, \lg A$ and $\lg M$ in patients with oral lichen ruber. Coll Antropol. 2008;32:161-3

54. Phillips AC, Carroll D, Drayson MT, Der G. Salivary Immunoglobulin A Secretion Rate Is Negatively Associated with Cancer Mortality: The West of Scotland Twenty-07 Study. PLoS One. 2015;23(10):e0145083.

55. Garcia-Pola MJ, Llorente-Pendás S, Seoane-Romero JM, Berasaluce MJ García-Martín JM. Thyroid Disease and Oral Lichen Planus as Comorbidity: A Prospective Case-control Study. Dermatology. 2016;232:214-9.

56. Larsen KR, Johansen JD, Reibel J, Zachariae C, Pedersen AM. Symptomatic oral lesions may be associated with contact allergy to substances in oral hygiene products. Clin Oral Investig. 2017;13. doi:10.1007/s00784-017-2053-y.

\section{Submit your next manuscript to BioMed Central and we will help you at every step:}

- We accept pre-submission inquiries

- Our selector tool helps you to find the most relevant journal

- We provide round the clock customer support

- Convenient online submission

- Thorough peer review

- Inclusion in PubMed and all major indexing services

- Maximum visibility for your research

Submit your manuscript at www.biomedcentral.com/submit
) Biomed Central 